\title{
Spare Parts Demand Prediction Research of Navigation Marks Based on the Method of Quadratic Exponential Smoothing
}

\author{
Wen Quan \\ Changjiang Waterway Planning Design and Research Institute, Wuhan, China
}

58071899@qq.com

\begin{abstract}
This paper aims to research and provide a reasonable prediction method that can be applied on routine work of navigation marks. On one hand, the reasonable prediction method can help maintenance and management unit to reduce cost and relative risk. On the other hand, according to Yangtze river waterway practical condition and historical consumption data of navigation marks' spare parts, the study has shown the quadratic exponential smoothing method be adopted in actual consumption research can satisfy the requirement of demand prediction.
\end{abstract}

Keyword: Quadratic exponential smoothing, Navigation mark, Spare parts, Prediction

\section{Introduction}

Navigation mark frequently set in inland river and offshore bank. Meanwhile, such as crossing mark, bankwise mark, leading mark, lateral mark, separate channel mark, surface emergency position-indication radio beacon(S-EPIRB) and bridge opening mark are collectively referred to as navigation mark. Similarly, navigation marks are widely adopted in Yangtze river for navigation safety demand. On the basis of this main reason, the spare parts and materials' securely supply of navigation marks have increasingly became more and more important. At the same time, navigation mark is made by lots of different components and also need a larger number of accessories to keep its on normal working condition. For the sake of low cost, low inventory and accurate management of different spare parts and accessories, the scientific and reasonable demand prediction research should be applied on routine work of navigation mark.

\section{Literature Review}

The mathematical method of exact demand prediction was always understanding as a key research issue in amount of management fields that include raw material purchase of enterprises, law of consumers demand, reasonable inventory management and so on. In order to achieve the several advantages of low cost, low inventory and low risk in market competition and reasonable management, a suitable and exact mathematical method of demand prediction can be used to promote core competitiveness and reduce associated risks in operation.

Jain $^{[1]}$ researches on 1,236 companies' forecast models and results to an obvious conclusion that 61.33\% adopt time series analysis method, $22.65 \%$ adopt causal method, $13.92 \%$ adopt judgement analysis method and rest of $2.1 \%$ companies use other prediction methods. According to those research data, most of companies in routine operation have became more and more concentrate on influence of demand by external multi-factors. In the study areas of demand prediction, researchers provide a lot of useful results that applied in different field. Gardner ${ }^{[2]}$ in his article states the research situation of exponential smoothing method, meanwhile, its presents the economic prediction characteristic, robust characteristics and some limitations of this method. Thomassey and 
Happiette $^{[3]}$ base on neural network and applied clustering and classification tool to provide an assistance decision making system to work out the products' medium-term sales forecast problem which is lack of historical sales data. Chern ${ }^{[4]}$ gives a new product sales forecast system and try to solve the problem of new product sales forecast, in terms of results that this system was given by him is better than moving average method under a small amount of subjective factors involved. Jeong ${ }^{[5]}$ bases on canonical genetic algorithm(CGA) and guided genetic algorithm(GGA) to build a deterministic parameters’ linear regression model.

\section{Navigation marks' spare parts demand in Yangtze river}

Yangtze river is the longest inland river in China and is known as "Golden Waterway" by worldwide. In order to satisfy the ever-growing freight transport demand, meanwhile, ensure and construct an efficient, safe and green waterway in maintenance and management. Those aims as mentioned above, its obviously certify a key point that normal operation of navigation marks were greatly important for waterway demand ${ }^{[6]}$. Actually, the navigation mark is made by solar panel, storage battery, pedestal, wire, remote terminal unit and so on. Moreover, the Yangtze river waterway maintenance and management units simultaneously provide a series of other materials for normal operation demand of navigation mark. Currently, the steel wire rope, cone, anchor, cement anchor, tank and lightship are also frequently-used by maintenance and management work. This paper selects the consumption of steel wire rope as study sample that is widely used and the biggest consumption in routine work. According to historical data that collection in waterway maintenance and management unit, the figure 1 shows the monthly consumption of steel wire rope as follow, and the monthly average consumption is 1660 meters.

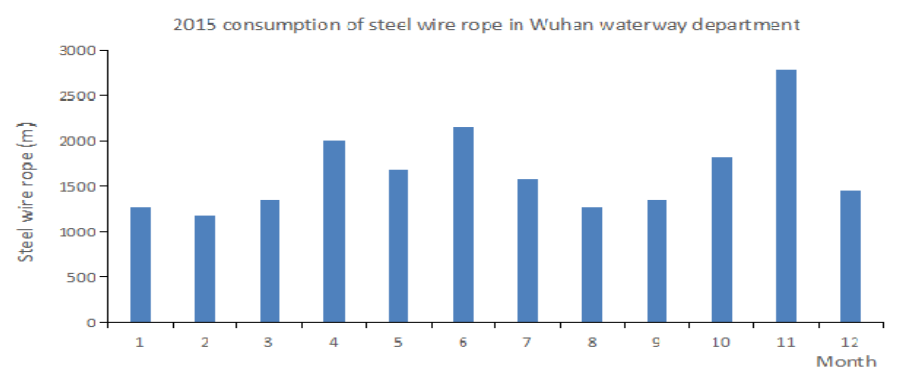

Figure 1. 2015 consumption of steel wire rope in Wuhan waterway department

Compare with the method of regression analysis, the method exponential smoothing usually adopted in forecast research that bases on a series of historical data analysis to forecast the data change in the future.

Single exponential smoothing forecast mainly concentrates on previous actual data and weighted average of predicted value, this method was usually adopted by no obvious change of trend and season's time series. However, in the actual research, this paper has to take into account of trend and season change.

On account of water level seasonal changing in Yangtze river, the number of navigation marks are changing by layout requirement and the quantity of navigation ships also influenced during flood season and dry season. Obviously, the influence factors of trend value and seasonal fluctuation should be introduced in wire rope of navigation marks prediction research. Therefore, single exponential smoothing forecast can be unsuited for navigation marks' spare parts' prediction research $^{[7-8]}$.

For research demand, this paper aims to modify the single exponential smoothing forecast and add a new smoothing factor to mathematical model as follow: 


$$
\begin{aligned}
& \hat{X}_{t+1}=\alpha X_{t}+(1-\alpha)\left(\hat{X}_{t}+T_{t}\right) \\
& T_{\mathrm{t}+1}=\beta\left(\hat{X}_{\mathrm{t}+1}-\hat{X}_{\mathrm{t}}\right)+(1-\beta) T_{t} \\
& Y_{\mathrm{t}+1}=X_{\mathrm{t}+1}^{\wedge}+T_{\mathrm{t}+1}
\end{aligned}
$$

As the formula mentioned above:

$Y_{\mathrm{t}+1}$----After $\mathrm{t}+1$ period of calibration trend prediction

$\hat{X}_{\mathrm{t}+1}$------After $\mathrm{t}+1$ period of initial trend

$T_{\mathrm{t}+1}$----Trend of $\mathrm{t}+1$ period

$\alpha$----The smoothing coefficient $(0 \leq \alpha \leq 1)$

$\beta$---- Trend of smoothing coefficient

Firstly, this paper assumes the average consumption value of last year is previous forecast value and the trend change impacts on the value of $\alpha$. Meanwhile, in research $\alpha$ and $\beta$ are respectively assigned a value of 0.3 and 0.4 . Consequently, the calculation results of single exponential smoothing forecast and quadratic exponential smoothing forecast clearly shows in following table 1 and figure 2.

Table 1. Calculation results of single exponential smoothing forecast and quadratic exponential smoothing forecast

\begin{tabular}{cccc}
\hline Month/Year & 2015 & $\begin{array}{c}\text { Single exponential } \\
\text { smoothing forecast(2016) }\end{array}$ & $\begin{array}{c}\text { Quadratic exponential s } \\
\text { moothing }\end{array}$ \\
\hline 1 & 1270 & 1600 & 1576 \\
2 & 1190 & 1675 & 1631 \\
3 & 1350 & 1723 & 1676 \\
4 & 2015 & 1774 & 1731 \\
5 & 1680 & 1757 & 1704 \\
6 & 2160 & 1756 & 1695 \\
7 & 1580 & 1732 & 1664 \\
8 & 1275 & 1734 & 1657 \\
9 & 1345 & 1762 & 1668 \\
10 & 1820 & 1794 & 1676 \\
11 & 2780 & 1802 & 1658 \\
12 & 1460 & 1748 & 1588 \\
\hline
\end{tabular}




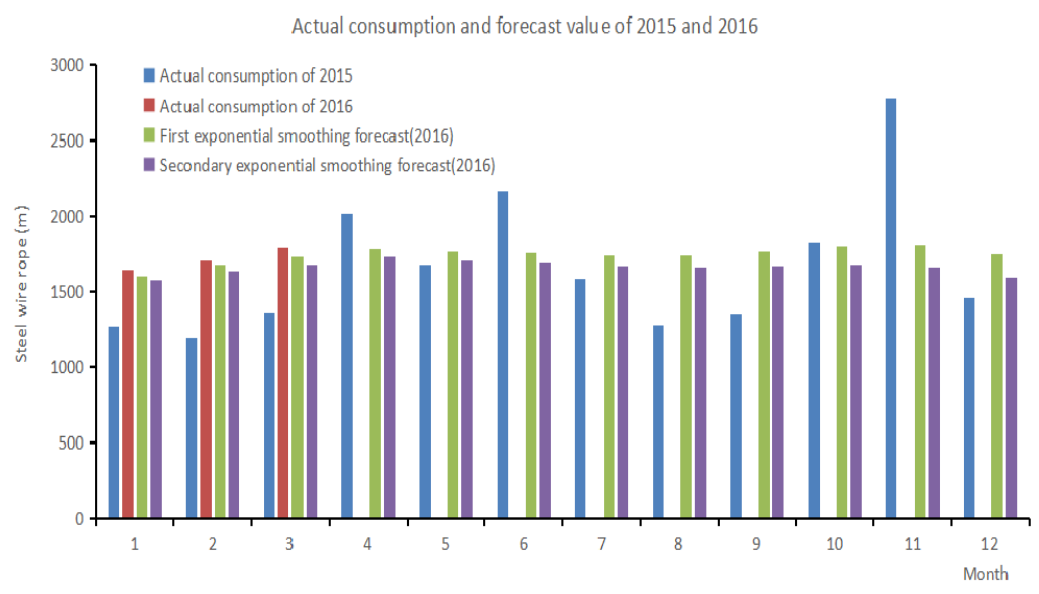

Figure 2. Actual consumption and forecast value of 2015 and 2016

According to those results and actual consumption of the first 3 months of 2016 mentioned above, this paper research results exist a certain error on future forecast, therefore, a formula should be introduced to amend the predict loss of degree of freedom and correct a parameter of $\alpha$ in previous formula.

$$
S_{F}=\sqrt{\frac{\sum_{t}(X t-Y t)^{2}}{N-1}}
$$

As the formula mentioned above:

$S_{F}$----Standard error of prediction

$X_{t}$----Actual demand of period $\mathrm{t}$

$Y_{t}$----Predicted value of period $\mathrm{t}$

\section{$N$----The number of forecast period $\mathrm{t}$}

This paper assumes the value of demand forecast obeys normal distribution and set 95\% confidence interval of third month and fourth month demand in 2016, consequently, in terms of formula (3-4) can obtains two values of standard error equal 94.11 and 101.83.

Lookup table as research knows that $Z_{\alpha=0.95}=1.96$, therefore, that the optimum prediction of actual demand in third month and fourth month of 2016 as follow:

$$
\begin{aligned}
F_{3} & =Y_{3} \pm Z_{\alpha=0.95}\left(S_{F}\right) \\
& =1676 \pm 1.96 * 94.11 \\
& =1676 \pm 184 \\
F_{4} & =Y_{4} \pm Z_{\alpha=0.95}\left(S_{F}\right) \\
& =1731 \pm 1.96 * 101.83 \\
& =1731 \pm 200
\end{aligned}
$$

Therefore, the actual demand(F) in fourth month of prediction in the $95 \%$ confidence interval are $1492 \leq F_{3} \leq 1860$ and $1531 \leq F_{4} \leq 1931$, meanwhile, compare with those forecast values that display an obvious result the method of quadratic exponential smoothing forecast is better than single 
exponential smoothing forecast in this study and prediction results are more close to the actual demand and historical data.

\section{Conclusion}

This paper concentrates on the consumption problem of navigation marks' spare parts and analyses a series of historical data to known its internal relations and law, meanwhile, the two influence factors are considered in study and research provides an obvious result that method of quadratic exponential smoothing forecast is fit for demand forecast in navigation marks' spare parts research. At last, the theory of confidence interval is also introduced in study to promote the range of forecasting numerical and help operators to face actual working situation. In the meantime, in order to satisfy some demand fluctuation suddenly, the multi-factors influence should be considered in research method in future study.

\section{Reference}

[1]Jain C L. Bench marking forecasting models[J].The Journal of Business Forecasting Methods and System,2002,21(3):18 - 20.

[2]Gardner J E S.Exponential smoothing:The state of the art-Part II[J].International Journal of Forecasting,2006,22( 4) :637 - 666.

[3]Thomassey S,Happiette M. A neural clustering and classification system for sales forecasting of new apparel items[J]. Applied Soft Computing,2007,7( 4) :1177-1187.

[4]Chern C C, Wu L L, Kung L C. Designing a decision-support system for new product sales forecasting[J]. Expert Systems with Applications,2010,37( 2) :1654-1665.

[5]Bates J N,Granger C W J. Combination of forecasts[J]. Operations Research Quarterly,1969,(4) :451-468.

[6]Jeong B,Jung H J,Spark N K. A computerized causal forecasting system using genetic algorithms in supply chain management[J]. Journal of Systems and Software,2002,60(3) :22 -237.

[7]Chandra C,Grabis J. Application of multi-steps forecasting for restraining the bullwhip effect and improving inventory performance

under autoregressive demand[J]. European Journal of Operational Research,2005,166( 2) :337-350.

[8]Liang W Y,Huang C C. Agent-based demand forecast in multi-echelon supply chain[J]. Decision Support Systems,2006,42( 1) :390-407. 